\title{
Mathematical and Computational Modeling of Neurons and Neuronal Ensembles
}

\author{
Andreas Schierwagen \\ Institute for Computer Science, Intelligent Systems Department, \\ University of Leipzig, Leipzig, Germany \\ schierwa@informatik.uni-leipzig.de \\ http://www.informatik. uni-leipzig.de/ schierwa
}

\begin{abstract}
In Computational Neuroscience, mathematical and computational modeling are differentiated. In this paper, both kinds of modeling are considered. In particular, modeling approaches to signal generation and processing in single neurons (i.e., membrane excitation dynamics, spike propagation, and dendritic integration) and to spatiotemporal activity patterns in neuronal ensembles are discussed.
\end{abstract}

Keywords: neurons, neuronal ensembles, mathematical, computational, modeling.

\section{Introduction}

Two kinds of modeling are differentiated in Computational Neuroscience (CNS), mathematical and computational modeling. The first emerges from applying mathematics to neuroscience in the way that is standard in science. This conventional modeling concentrates on the analysis of structure and dynamics of the brain and its parts. Thus it is confined to the nervous system itself. The other kind, computational modeling, is concerned with function, which in the classical AI tradition means, information representation, processing and manipulation, learning and decision-making. Alternative directions think of function rather in terms of behavior, e.g. visual scene analysis, sensomotor coordination, reaching and grasping, and navigation. Here modelers are forced to deal with an organism embedded in an environment. There are several features common to both modeling kinds: they require an abstraction process by which presumably negligible details are eliminated. Any instance of a computational model is a mathematical model, and the two are normally closely meshed with one another in particular research subjects. In principle, by this meshing computational constraints could guide the appropriate design of mathematical models, but these techniques are not yet widely used. In the following, both kinds of modeling in CNS are considered. In particular, modeling approaches to signal generation and processing in single neurons and to spatiotemporal activity patterns in neuronal ensembles are discussed. 


\section{$2 \quad$ Single Neuron Modeling}

At the level of single neuron modeling, the cable theory of signal spread in passive dendrites, the Hodgkin-Huxley model relating action potential, ionic conductances and membrane particles, and the compartmental modeling approach to complex branched neurons represent the "working horses" of CNS [1. Two types of complexity must be dealt with: the intricate interplay of active conductances underlying the complex neuronal excitation dynamics, and the elaborate dendritic morphology that allows neurons to receive and process inputs from many other neurons (e.g. 2]). Their specific morphology is used to classify neurons 1 (Fig. 1).

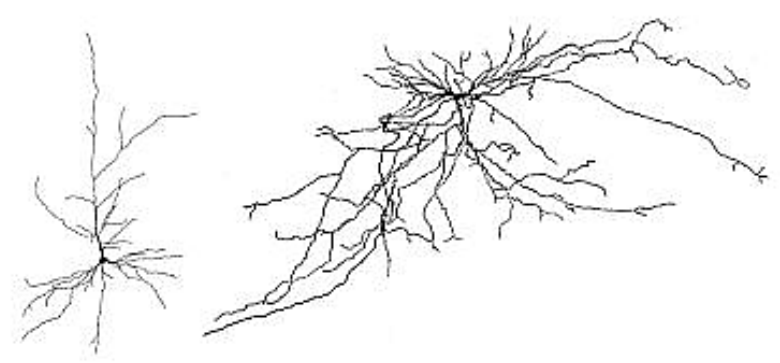

Fig. 1. Examples of rendered neuron morphologies. Left: pyramidal neuron of the mouse somatosensory cortex [4, Right: wide-field neuron of the cat superior colliculus [5].

\subsection{Mathematical Models}

Membrane excitation dynamics. The evolution of the membrane potential of a neuron is described by a system of coupled, non-linear ordinary differential equations, such as the Hodgkin-Huxley model [6]. The Hodgkin-Huxley model is well beyond analytical solution; fortunately, dynamical systems theory provides insights into how neuron activity is shaped by individual neuronal parameters, such as the maximal conductance of a particular membrane current. For instance, in [7], the functional role of the repolarizing ionic currents in the periodic activity of nerve membranes was analyzed. The Hodgkin-Huxley equations can be driven into repetitive activity by a maintained depolarizing current $I$, a decrease in the maximal $K^{+}$-conductance $g_{K}$, or by moving the Nernst potential $V_{K}$ for $K^{+}$in the depolarizing direction. In all these cases large amplitude periodic solutions are obtained (Fig. 2, left). Changing two parameters gives rise to bifurcation curves in the parameter plane. For the parameters $I$ and $V_{K}$ a region of multiple equilibria is found. The three equilibrium solutions occur in the interior of the solid curves in Fig. 2 (right) where the dashed curve represents the Hopf bifurcation.

\footnotetext{
${ }^{1}$ For a review of our approaches to morphological quantification and mathematical
} modeling of neuron growth and structure, see [3]. 

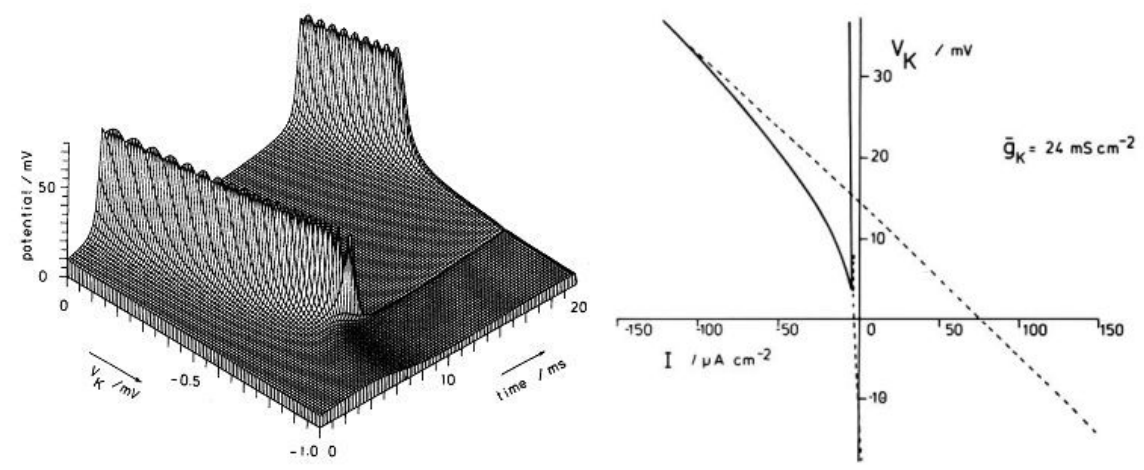

Fig. 2. Left: Numerical solutions of the standard Hodgkin-Huxley membrane equations as $V_{K}$, the Nernst potential for $K^{+}$, is changed.. Right: Bifurcation curve in the $I-V_{K}$ plane at a specific value of $g_{K}$ 7]. See text for details.

Models of dendritic integration. Models of the integrative neuron function differ in the extent to which the branching geometry of the dendrites is considered. Originally, the cable equation describing passive membrane voltage changes and current spread was assumed to be linear 2 , and the complex morphology was reduced to a single, electrically equivalent cylinder (EC) 8]. It turned out, however, that most of the restrictions imposed by Rall's EC model are not fulfilled in reality [9, and the model has lost the favorite state for the interpretation and prediction of experiments. In order to overcome this, two alternatives have been followed: a) to modify the EC model and b) to implement a compartmental model.

In [10, the main types of passive dendritic cable models, including the equivalent cable (ECa) model were presented. The ECa model is based on the cable equation with spatially varying parameters. While this equation can be solved in general only numerically, we were able to formulate a branching condition (comprising the idealized geometry necessary for the EC model) under which analytical solutions can be deduced (Fig. 3, left), and branching patterns found in dendritic neurons could be analytically treated [11|12].

Based on experimental data of several types of neurons, compartment models have been used for exploring intraneuronal signal processing, and to analyze the impact of dendritic morphology and non-uniform ion channel distribution on neuron function. These models can be employed in two ways: to solve the inverse problem (i.e., to determine membrane parameters) and to do forward calculations [13]. The inverse problem was shown to be ill-posed, i.e. parameter estimation is not unique. Using physiological restrictions, several admissible parameter combinations can be determined. In the forward calculations, a model

\footnotetext{
${ }^{2}$ Now it is unquestionable that many if not most dendrites are not passive but active, and thus the nonlinear cable equation or a corresponding compartmental model must be used.
} 


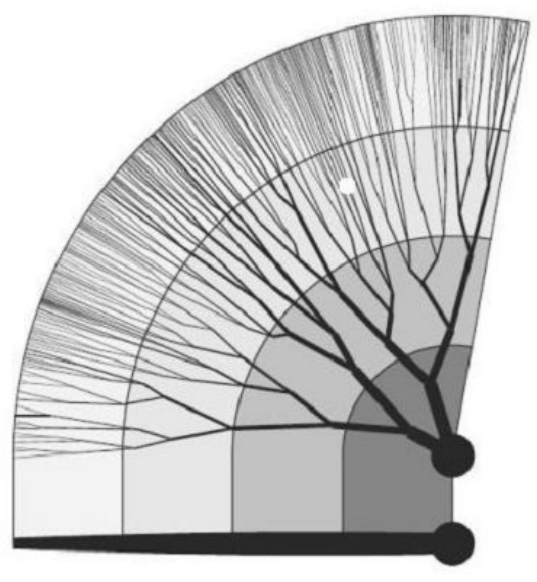

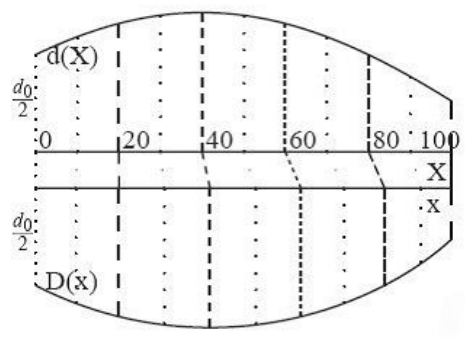

Geometry type: sine/cosine

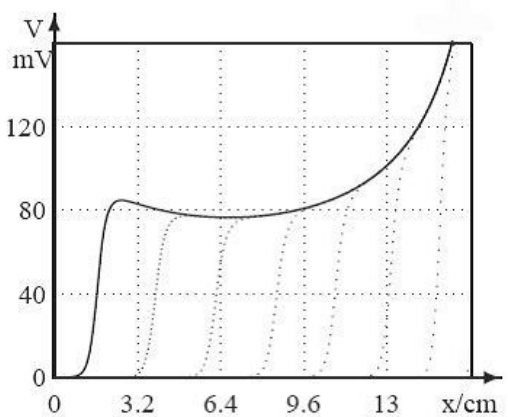

Fig. 3. Left: Equivalent cable model. The reduction of a neuron with two stem dendrites to an equivalent cable is illustrated. The lines connect points of equal electrotonic distance on the dendrites and on the nonuniform equivalent cable with sine/cosine profile. Right: Propagation of excitation front along a neurite of the sine/cosine geometry type. Displayed is the neurite diameter function in anatomical and electrotonic space (top), and snapshots of an excitation front moving leftwards (bottom). Notice that amplitude changes with diameter. After [12].

with fitted parameters is applied to calculate functional characteristics of, e.g., passive dendrites such as attenuation, delay and time window of synaptic input signals. In this way, clues for the possible function of the neurons studied can be derived. For example, neurons from superior colliculus (a part of the midbrain) could be differentiated w.r.t. to computing function as coincidence detectors and integrators, respectively [5] (see below, paragraph 2.2).

In the study [4], we employed compartmental modeling to perform a comparative electrotonic analysis of two samples of cortical pyramidal neurons, one from wildtype, and the other from transgenic mice. While anatomical dendritic trees of transgenic pyramidal neurons were significantly enlarged, the statistical analysis of the sample morphoelectrotonic (MET) dendrograms revealed that the transgenic neurons scaled in a MET-conserving mode. This means, the dimensions of their dendritic trees changed in such a way that direction- and frequency-dependent signal propagation in the passive neuron models was little affected! 
The nerve conduction model. For the conduction of nerve impulses along a neurite (dendrite or axon), the model involves a nonlinear parabolic partial differential equation (PDE) such as those of Hodgkin and Huxley $[\underline{6}$ or FitzHugh and Nagumo (14]). Its solutions mimic membrane potential and current as a function of time and distance along the neurite.

In the standard case of neurites with uniform electrical and geometric properties, a traveling wave solution exists, and an explicit velocity-diameter relationship for nerve fibers can be given. Experimental effects which could not be explained with this theory include blocking of impulse conduction and changes of action potential (AP) shape in regions of nonuniform axon geometries (for review, see [15]). Using the FitzHugh-Nagumo PDE, we could show that AP propagation in a non-uniform neurite is equivalent (under certain variable transformations) to the homogeneous case 14]16. The transformation conditions determine six specific neurite geometries enabling exact solutions, including diameter profiles of the type power, exponential and sine/cosine function. For these inhomogeneous neurite geometries, explicit formulas were derived reproducing the observed relationship between neurite geometry type and AP shape, velocity and frequency [14]16] (see Fig. 3, right).

\subsection{Single-Neuron Computations}

Computing in computer science means implementing an algorithm, that is, a sequence of simple computational steps that map the input to the output. Based on this model, ways are searched for decomposing into such simpler building blocks the very complex mapping done by a neuron, and for determining the units of computation. At the level of single neurons, literally each structural part, i.e. dendrites, spines, cell body and axon, has been considered as possible functional unit:3. For example, models of dendritic neurons have been used to implement Boolean logical operations, to compute the movement direction of a stimulus, and to simulate coincidence detection in auditory neurons (see [18) for review). The possible computational functions of axons have been less studied. The nonlinear interactions (observed and modeled, see above, paragraph 2.1) of action potentials at regions of changing axon geometry could serve computational functions. E.g., a reduction of spike frequency at branching points [15]) could be exploited in brain networks using rate coding.

Summing up, there is no doubt that single neurons dispose of a range of mechanisms that could be used to implement elementary computations. Proving that neurons, dendrites etc. do really a specific computation is not possible, even if this has been claimed. In the cases mentioned (and in general) only indirect evidence is available or can be expected, due to the problems that inhere in the computational approach itself [19].

\footnotetext{
3 The underlying concept of decompositional brain analysis has been critiziced in 17. There I concluded that in complex systems like the brain, structural and functional components generally do not match up one-to-one.
} 


\section{$3 \quad$ Modeling Neural Ensembles}

The distributed activity of neural ensembles, i.e. large populations of neurons, in the form of, e.g. oscillations and traveling waves, is known to play an important role in the nervous system. A common starting point for analyzing the largescale dynamics of cortex is to treat nerve tissue as a continuous two-dimensional medium, so-called neural fields.

\subsection{Neural Fields and Their Dynamics}

The work of Amari [20] has provided a categorization of the dynamics of onedimensional, homogeneous neural fields with symmetrical lateral coupling functions. In one-layer fields, five types of dynamics were proved to exist, which are in general multi-stable. Among them are stationary, localized excitation regions, often referred to as bumps, and several modes of interaction of excitation regions. Two-layer fields admit oscillatory and traveling wave solutions. These results transfer to two-dimensional neural fields, but new types of dynamics appear $[21|22| 23]$.

Using computer simulations [24, we found that inhomogeneous neural fields with asymmetrical coupling functions can produce stable bumps moving on the neural field (Fig. 4).

\subsection{Analog Computations in Neural Fields}

The rich dynamic behavior of neural fields has been successfully employed to realize analog computations. This has been based on the idea of mapping a particular problem to be solved onto the dynamics of a neural field. The problem solution can be obtained then by following the spatiotemporal field evolution.

In general, homogeneous, symmetrically connected networks have been used as models of neural computations. However, biological neural networks have asymmetrical connections, at the very least because of the separation between excitatory and inhibitory neurons in the brain. It has been shown that the distinctly different dynamical behaviors they present can make the asymmetrical networks computationally beneficial 24]. In [25], we proposed a neural field model of dynamic control of fast orienting eye movements (saccades). The model realizes the short-term memory of target location using a homogeneous field with symmetrical couplings, and the dynamic motor error coding via the hill-shift effect in an inhomogeneous field with asymmetrical couplings. The different schemes of lateral coupling have been chosen in general agreement with experimental findings. Fig. [- shows the modeled hill-shift effect as found in the superior colliculus of the cat.

From a general point of view, the interpretation in terms of computations of the activity patterns appearing in neural fields can be easier achieved, as compared with single neurons. This is due to the experimentally available techniques which can be used to demonstrate correlations between recorded activity 

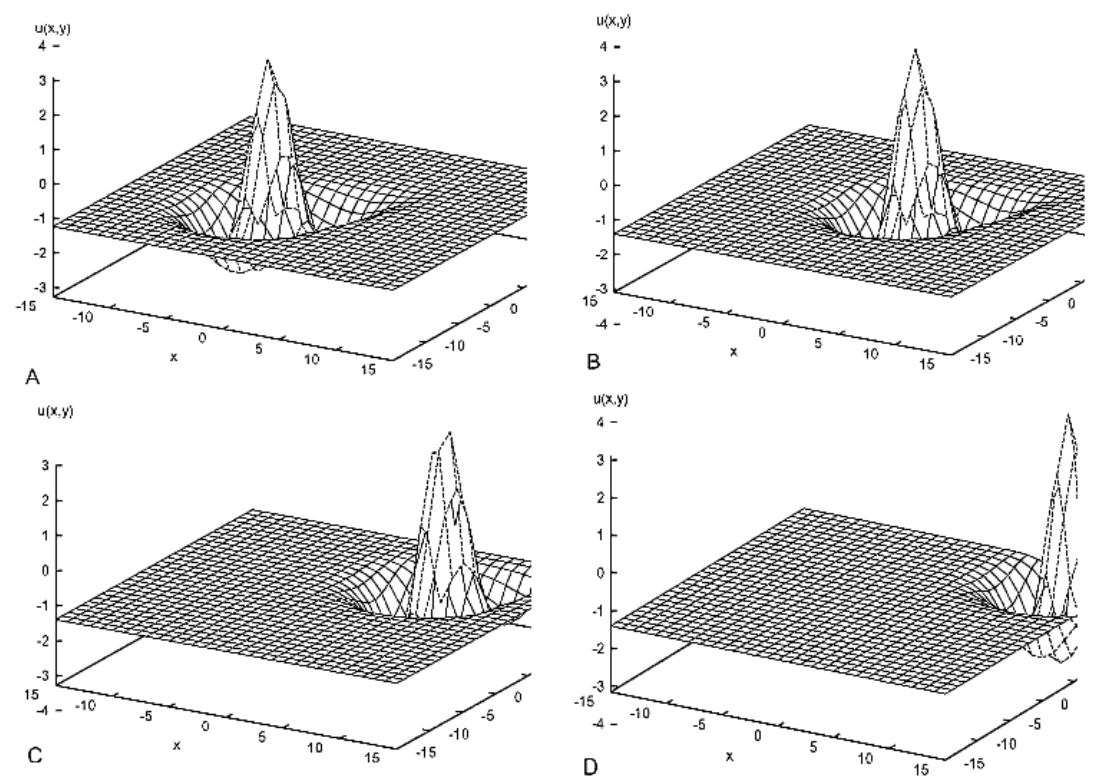

Fig. 4. Hill-shift effect in an inhomogeneous field with asymmetrical couplings. The bump moves from start location (A) via (B), (C) to location (D), see [24].

patterns and behavior of the anima 4 . Because of the nature of the modeling relation [17] for complex systems like the brain, an ultimate proof that a particular computation produces a particular behavior is not possible.

\section{References}

1. Koch, C., Segev, I. (eds.): Methods in Neuronal Modeling. From Ions to Networks. MIT Press, Cambridge (1998)

2. Schierwagen, A.: Growth, structure and dynamics of real neurons: Model studies and experimental results. Biomed. Biochim. Acta 49, 709-722 (1990)

3. Schierwagen, A.: Neuronal morphology: Shape characteristics and models. Neirofiziologiya/Neurophysiology 40, 366-372 (2008)

4. Schierwagen, A., Alpár, A., Gärtner, U.: Scaling properties of pyramidal neurons in mice neocortex. Mathematical Biosciences 207, 352-364 (2007)

5. Schierwagen, A., Claus, C.: Dendritic morphology and signal delay in superior colliculus neurons. Neurocomputing 38-40, 343-350 (2001)

6. Hodgkin, A.L., Huxley, A.F.: A quantitative description of membrane current and its application to conduction and excitation in nerve. J. Physiol. 117, 500-544 (1952)

${ }^{4}$ At the level single neurons or below, such correlations with animal behavior cannot be seriously expected. 
7. Holden, A.V., Muhamad, M.A., Schierwagen, A.K.: Repolarizing currents and periodic activity in nerve membrane. J. Theor. Neurobiol. 4, 61-71 (1985)

8. Rall, W.: Theory of physiological properties of dendrites. Ann. N.Y. Acad. Sci. 96, 1071-1092 (1962)

9. Schierwagen, A.: Segmental cable modelling of electrotonic transfer properties of deep superior colliculus neurons in the cat. J. Hirnforsch 27, 679-690 (1986)

10. Schierwagen, A.: Exploring the computational capabilities of single neurons by continuous cable modelling. Prog. Brain Research 102, 151-168 (1994)

11. Schierwagen, A.: A non-uniform equivalent cable model of membrane voltage changes in a passive dendritic tree. J. theor. Biol. 141, 159-180 (1989)

12. Ohme, M., Schierwagen, A.: An equivalent cable model for neuronal trees with active membrane. Biol. Cybernet. 78, 227-243 (1998)

13. Schierwagen, A.: Identification problems in distributed parameter neuron models. Automatica 26, 739-755 (1990)

14. Schierwagen, A.: Impulse propagation in nonuniform fibres: analytical treatment. In: Fusco, D., Jeffrey, A. (eds.) Nonlinear Waves and Dissipative Effects. Pitman Research Notes in Mathematics, vol. 227, pp. 133-140. Longman, London (1991)

15. Waxman, S.G., Kocsis, J.D., Stys, P.K. (eds.): The Axon. Structure, Function and Pathophysiology. Oxford University Press, Oxford (1995)

16. Schierwagen, A., Ohme, M.: A Model for the Propagation of Action Potentials in Nonuniform Axons. AIP Proc. 1028, 98-112 (2008)

17. Schierwagen, A.: Brain Complexity: Anlysis, Models and Limits of Understanding. In: Mira, J., et al. (eds.) IWINAC 2009, Part I. LNCS, vol. 5601, pp. 195-204. Springer, Heidelberg (2009)

18. London, M., Häusser, M.: Dendritic computation. Ann. Rev. Neurosci. 28, 503-532 (2005)

19. Schierwagen, A.: Brain Organization and Computation. In: Mira, J., Álvarez, J.R. (eds.) IWINAC 2007. LNCS, vol. 4527, pp. 31-40. Springer, Heidelberg (2007)

20. Amari, S.: Dynamics of pattern formation in lateral-inhibition type neural fields. Biol. Cybernet. 27, 77-87 (1977)

21. Wellner, J., Schierwagen, A.: Cellular-Automata-like Simulations of Dynamic Neural Fields. In: Holcombe, M., Paton, R.C. (eds.) Information Processing in Cells and Tissues, pp. 295-304. Plenum, New York (1998)

22. Werner, H., Schierwagen, A.: Categorization of the dynamics in two-dimensional neural fields. In: Abstracts of the 9th Annual Computational Neuroscience Meeting, Brugge, Belgium, July 16-21, p. 156 (2000)

23. Werner, H., Richter, T.: Circular stationary solutions in two-dimensional neural fields. Biol. Cybern. 85, 211-217 (2001)

24. Schierwagen, A., Werner, H.: Analog computations with mapped neural fields. In: Trappl, R. (ed.) Cybernetics and Systems 1996, pp. 1084-1089. Austrian Society for Cybernetic Studies, Vienna (1996)

25. Schierwagen, A., Werner, H.: Fast orienting movements to visual targets: Neural field model of dynamic gaze control. In: 6th European Symposium on Artificial Neural Networks - ESANN 1998, pp. 91-98. D-facto publications, Brussels (1998) 